\title{
Two Dimension Numerical Simulation of Airflow over Complex Terrains
} at Low Altitude

\author{
Chu Tang, Huanran Hu, Guanxin Hong \\ School of Aeronautics Science and Engineering, Beihang University, 100191, China \\ E-mail: tangchu@ase.buaa.edu.cn
}

\begin{abstract}
In order to avoid the problems of existing methods, a numerical simulation method for two-dimensional airflow over complex terrains is developed in this paper for the engineering use of flight dynamics. Based on the potential flow theories, the effects of terrains on the wind field are considered by a serial of two-dimensional vortexes, whose strengths are solved by combining with the ground boundary conditions. Numerical examples are studied by the proposed method, and the method is also evaluated by comparing the results with ones from the existing method. The result shows that the two-dimensional profile of complex terrains could be described by a cubic spline curve precisely. The computation procedure proposed in this paper is very simple and efficient, and it could provide a result of wind field with considerable accuracy. Therefore, this method could be used for flight principle evaluation and flight simulators. Finally, through simulate flight path, discussing effect of terrains on track.
\end{abstract}

Keywords-component; complex terrains; airflow over mountain; cubic spline, potential flow theory style

\section{INTRODUCTION}

During take-off and landing, aircrafts are always affected by the atmospheric disturbances at low-altitude. The disturbances will also have a great influence on the flight characteristics of aircrafts that are executing the missions like low-altitude manned penetration. The techniques of wind field modeling and simulation are very important for both of the flight safety and flight dynamic response analysis. As the complex terrain is the main reason that induces disturbances at low-altitude, and it is also an important element to establish the low-altitude flight environment in a simulator, so it is necessary to develop a numerical method for the low-altitude wind field simulation which could consider the influence of complex terrains.

There are mainly three different types of low-altitude wind field simulation methods at present. The first one is developed by the atmospheric dynamics theory, which could consider the variation of atmospheric parameters, such as the temperature, energy and pressure. Terry L. Clark [1] and Peng $\mathrm{Hu}$ [2] simulate the three-dimensional airflow over mountains by using this method, then compare the results with ones from observation and wind tunnel test and get a good match. However, its computation progress is very complex, which is not suitable for the engineering application of flight dynamics analysis. The second one is the engineering simulation method based on the potential flow theory of fluid dynamics. The assumption of the ideal gas has been adopted, and the temperature variation has been neglected. Guanxin Hong [3] and Gang Liu [4] simulate the two-dimensional airflow over the mountains by the airflow over a cylinder, which could be represented by a fundamental solution of potential flow, one of the streamlines has been chosen as the shape of the terrains. It has a simple process, and could describe the airflow over mountains clearly. But there are only limited kinds of mountain shapes, and need a lot of manual parameter adjust works. The last one is the table lookup method, which means all the data is measured and stored in the computer. It is close to practice, but requires a huge amount of data storage space, and costs a lot of labor and resources.

Based on the potential flow theory of aerodynamics and the boundary conditions of terrain shapes, a numerical simulation method has been proposed according to the engineering application requirements. This method could simulate the wind field at low-altitude that influenced by arbitrary terrain profiles. Moreover, numerical examples have been studied, and the results are compared with the ones mentioned in [3] and [4].

\section{THEORY}

Wherever Times is specified, Times Roman or Times New Roman may be used. If neither is available on your word processor, please use the font closest in appearance to Times. Avoid using bit-mapped fonts if possible. True-Type 1 or Open Type fonts are preferred. Please embed symbol fonts, as well, for math, etc.

\section{A. Complex terrain modeling}

The Cartesian coordinate system $O x y$ is used to describe the terrain shape and wind field, where, the origin $O$ is an arbitrary point, the $x$ axis represents the horizontal distance, and the $y$ axis represents the altitude. For the twodimensional cases, the complex terrains could be represented by a complex curve in plane $O x y$ (as shown in Fig. 1). In this paper, the terrain curve is simulated by a cubic spline curve. If the cubic spline curve passes through a number of predefined points $\left(x_{i}, y_{i}\right)$, where, $j=1,2, \ldots, m$, and $m$ is the number of the points. Then the cubic spline curve could be described by a serial of third order polynomials 


$$
\begin{gathered}
y=a_{j} x^{3}+b_{j} x^{2}+c_{j} x+d_{j} \\
\left(x \in\left[x_{j}, x_{j+1}\right], j=1,2, \cdots, m-1\right)
\end{gathered}
$$

where, $a_{j}, b_{j}, c_{j}, d_{j}$ are the fitting coefficients of the $j$ th section. All of the coefficients could be solved by using the coordinates of the predefined points and the continuity conditions of cubic spline curve.

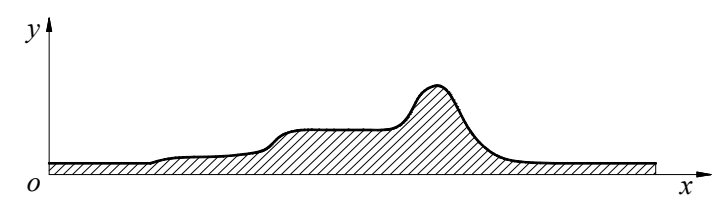

Figure 1. Complex terrains in the reference coordinate system

\section{B. Wind field modeling}

Similarly to the solving approach of vortex lattice method in aerodynamics, the curve that describes the terrain is divided into a number of straight line elements, and the number is $n$. A two-dimensional vortex is arranged at the quarter point of each element. Choose the three-quarter point of the element as the colocation point. At this point the actual boundary condition is implemented. Therefore, the effect of the terrains on the wind field could be represented by the sum of induce velocities of all the vortexes at any arbitrary point in the wind field. Use the uniform free stream to represent the wind field without terrains, the stream function is,

$$
\psi_{0}=-v_{0} x+u_{0} y
$$

where, $u_{0}$ and $v_{0}$ are two components of the uniform free stream velocity $\boldsymbol{V}_{\infty}$, and $\boldsymbol{V}_{\infty}=\left[u_{0} v_{0}\right]^{\mathrm{T}}$.

For the $i$ th element, assume that the vortex strength is $\Gamma_{i}$. The coordinates of vortex is $\left(x_{v j}, y_{v j}\right)$, which could be obtained by cubic spline function eq.(1), also does the coordinates $\left(x_{c j}, y_{c j}\right)$ and normal vector $\boldsymbol{n}_{i}$ of the collocation point. Based on potential flow theory, the stream function of $i$ th vortex is

$$
\psi_{i}=-\frac{\Gamma_{i}}{2 \pi} \ln \sqrt{\left(x-x_{\mathrm{v} i}\right)^{2}+\left(y-y_{\mathrm{v} i}\right)^{2}}
$$

where, $P(x, y)$ is an arbitrary point in the wind field. Therefore the velocity that induced by the $i$ th vortex at point $P$ could be expressed as

$$
\begin{gathered}
u_{\mathrm{P} i}=-\frac{\left(y-y_{\mathrm{v} i}\right)}{2 \pi r^{2}} \Gamma_{i} \\
v_{\mathrm{P} i}=-\frac{\left(x-x_{\mathrm{v} i}\right)}{2 \pi r^{2}} \Gamma_{i}
\end{gathered}
$$

where, $r=\left[\left(x-x_{v i}\right)^{2}+\left(y-y_{v i}\right)^{2}\right]^{1 / 2}$ is the distance between $P$ and the $i$ th vortex. So the induced velocities of all the collocation points could be represented as

$$
\begin{aligned}
& \boldsymbol{u}=\boldsymbol{W}_{x} \boldsymbol{\Gamma} \\
& \boldsymbol{v}=\boldsymbol{W}_{y} \boldsymbol{\Gamma}
\end{aligned}
$$

where $\boldsymbol{u}, \boldsymbol{v}$ are the vectors that represent the induced velocity components of all the collocation points along the $x$ axis and $y$ axis, respectively; $\boldsymbol{W}_{x}, \boldsymbol{W}_{y}$ are their influence coefficients matrixes; and $\boldsymbol{\Gamma}$ is vortex strength vector of all the vortex elements.

To search for a singularity distribution that creates enclosed streamlines, the Neumann boundary condition [5] is used. For the collocation point of the $i$ th vortex element, there is

$$
\left(\boldsymbol{V}_{\infty}+\boldsymbol{V}_{i}\right) \boldsymbol{n}_{i}=0
$$

Where, $\boldsymbol{V}_{i}$ is the induced velocity at the $i$ th colocation point, $\boldsymbol{V}_{i}=\left[\begin{array}{ll}u_{i} & v_{i}\end{array}\right]^{\mathrm{T}} . \boldsymbol{n}_{i}$ is the normal vector at $i$ th element, $\boldsymbol{n}_{i}=\left[\begin{array}{ll}n_{x i} & n_{y i}\end{array}\right]^{\mathrm{T}}$. Equation (7) is expanding by (6),

$$
u_{i} n_{x i}+v_{i} n_{y i}=-u_{0} n_{x i}-v_{0} n_{y i}
$$

Express the boundary condition equations of all the collocation points by matrix,

$$
\boldsymbol{A}_{\mathrm{AIC}} \boldsymbol{\Gamma}=\boldsymbol{A}_{0}
$$

where, $\boldsymbol{A}_{A I C}$ is influence coefficient matrix of the normal induced velocity; $\boldsymbol{A}_{0}$ is constant coefficients vector that relative to the normal direction of the elements and the free stream velocity. Then the strength of the vortexes could be solved as

$$
\Gamma=A_{\mathrm{AIC}}^{-1} \boldsymbol{A}_{0}
$$

Superposition of the effects of uniform free stream and two-dimensional vortexes, the stream function of the wind field with complex terrains could be represented as

$$
\psi=\psi_{0}+\sum_{i=1}^{n} \psi_{i}
$$

The wind velocity at any arbitrary position could be solved by (10).

\section{MODEL VALIDATION}

Several numerical examples have been studied in this paper, including the examples that proposed by [3] and [4].

\section{A. Mountain shape simulation}

In [3], the airflow over a single mountain is simulated by the ideal flow around a cylinder. One of the streamline has been chosen as the shape of the terrains, a factor $S$ is used to get different mountain shapes. And [4] simulates the airflow over multiple mountains by superposition of the ideal flow around multiple cylinders. Because of the inherent property 
of the method, the kind of terrain shapes that could be modelled is limited. Moreover, all the parameters need manual adjustment, in order to get a much more approximate result of the mountain shape.

The flow around a cylinder could be solved by superposing two fundamental solutions of potential flow, which are the doublet and the uniform free stream. Write the speed of uniform free stream as $V_{\infty}$, which is along the $x$ axis. The number of doublets is $l$, coordinate of $i$ th doublet is $\left(x_{i}\right.$, $\left.y_{i}\right)$, and the radius of the relative cylinder is $R_{i}$. Therefore, the stream function of the wind field is,

$$
\psi=V_{\infty}\left[y-\sum_{i=1}^{l} R_{i}^{2} \frac{\left(y-y_{i}\right)}{\left(x-x_{i}\right)^{2}+\left(y-y_{i}\right)^{2}}\right]
$$

\section{B. Results comparison}

Set the position of the cylinder as $(0,-300)$, and $R=200 \mathrm{~m}$. Select the streamline passes through the point $(-\infty, 0)$ as the terrain shape. It could be expressed as

$$
\frac{\psi_{0}}{V_{\infty}}=y-R^{2} \frac{y+300}{x^{2}+(y+300)^{2}}
$$

Where, $\psi_{0}$ is stream function value, and the attitude of the mountain is $100 \mathrm{~m}$.

TABLE I. NUMERICAL RESULTS OF THE WIND VELOCITY THAT AFFECTED BY THE SINGLE MOUNTAIN

\begin{tabular}{|l|l|l|l|l|c|}
\hline \multicolumn{1}{|c|}{$\boldsymbol{x} / \boldsymbol{m}$} & \multicolumn{1}{c|}{$\boldsymbol{y} / \boldsymbol{m}$} & $\boldsymbol{V} / \boldsymbol{m} \boldsymbol{s}^{-\boldsymbol{I}}$ & $\boldsymbol{\theta}^{\boldsymbol{o}}$ & $\boldsymbol{\Delta} \boldsymbol{V}$ & $\boldsymbol{\Delta \theta}$ \\
\hline-2000 & 300 & 4.97 & 0.29 & $0.24 \%$ & $0.32 \%$ \\
\hline-1000 & 300 & 4.94 & 1.47 & $0.20 \%$ & $-2.71 \%$ \\
\hline 0 & 300 & 5.56 & 0.26 & $0.16 \%$ & - \\
\hline 1000 & 300 & 4.94 & -1.55 & $0.22 \%$ & $3.12 \%$ \\
\hline 2000 & 300 & 4.97 & -0.30 & $0.22 \%$ & $4.28 \%$ \\
\hline
\end{tabular}

The wind field is defined as $x \in[-3000,3000], y \in$ $[0,500]$. In this area, the terrain is divided into 100 elements. Set $\boldsymbol{V}_{\infty}=5 \mathrm{~m} / \mathrm{s}$. Fig. 2 shows the distribution of the wind velocity over the terrains, and Fig.3 shows streamlines calculated by the proposed method and the analytical solution of the flow around a cylinder. Viscous effects are both not considered. Table I lists the magnitude and direction of the wind velocities at several positions; it also lists the difference between the numerical results and the analytical results. Where, $(x, y)$ is coordinates of the position has been analysed, and $V$ is magnitude of the wind velocity, and $\theta$ is the angle between the $x$ axis and the wind velocity.

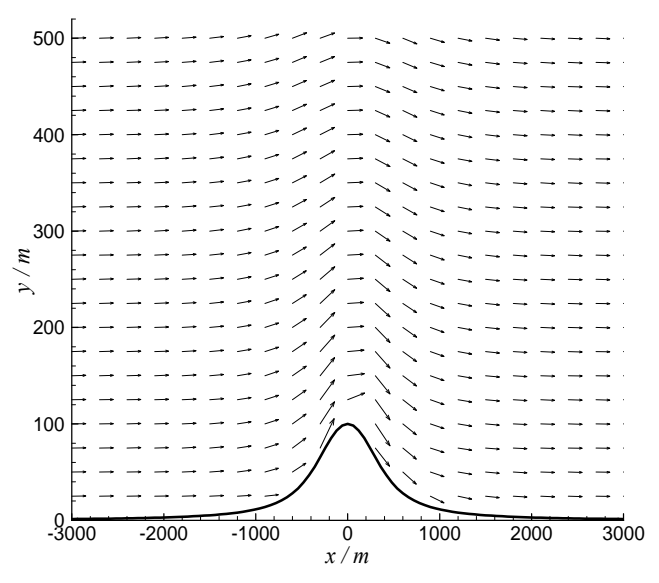

Figure 2. Wind velocity over the single mountain

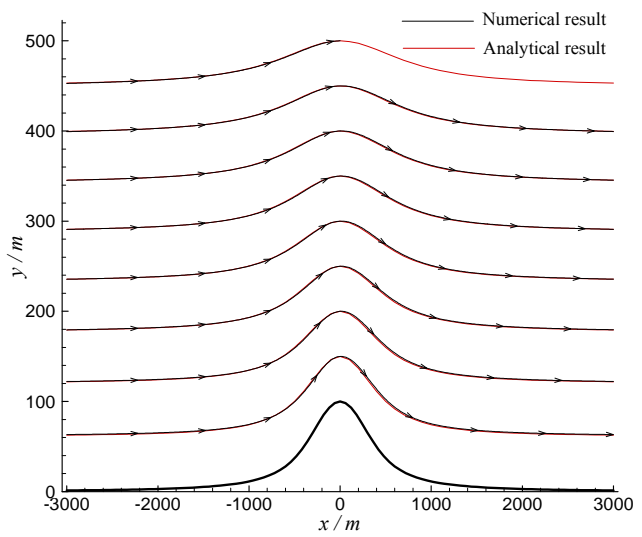

Figure 3. Streamline over the single mountain

Fig. 2 and Fig. 3 indicate that, the methods mentioned in [3] and [4] could only get the symmetrical terrain models. Compared with the analytical results, the numerical results obtained by the proposed method match it very well, the errors of the velocity magnitudes are all less than $1 \%$. And the absolute values of the error of the velocity direction are about $5 \%$.

\section{The results of airflow around multiple cylinders}

The position coordinates and geometric parameters of three cylinders are listed in table 2. Also choose the streamline that pass through the point $(-\infty, 0)$ as the terrain shape, then the streamline can be expressed as

$$
\frac{\psi_{0}}{V_{\infty}}=y-\sum_{i=1}^{3} R_{i}^{2} \frac{y-y_{i}}{\left(x-x_{i}\right)^{2}+\left(y-y_{i}\right)^{2}}
$$

Similarly, $\psi_{0}$ is the stream function value. It could be solved that the altitude of the three mountain are $h_{1}=110 \mathrm{~m}$, $h_{2}=170 \mathrm{~m}, h_{3}=210 \mathrm{~m}$, respectively 
TABLE II. POSITION AND GEOMETRIC PARAMETERS OF THE CYLINDERS

\begin{tabular}{|l|l|l|l|}
\hline \multicolumn{1}{|c|}{$\boldsymbol{x} / \boldsymbol{m}$} & \multicolumn{1}{c|}{$\boldsymbol{1}$} & \multicolumn{1}{c|}{$\boldsymbol{1}$} & \multicolumn{1}{c|}{3} \\
\hline$\left(x_{i}, y_{i}\right) / \mathrm{m}$ & $(-1500,-300)$ & $(0,-300)$ & $(1500,-300)$ \\
\hline$R_{i} / \mathrm{m}$ & 200 & 260 & 320 \\
\hline
\end{tabular}

The wind field is defined as $x \in[-5000,6000], y \in$ $[0,1000]$. Velocity of uniform free stream is $5 \mathrm{~m} / \mathrm{s}$. In this area, the curve of terrain is divided into 200 elements. Fig. 4 and Fig. 5 shows the results of wind velocity and streamlines over the multiple mountains. As same as table 1 , table 3 also lists the magnitude and direction of the wind velocities at several positions, it also lists the difference between the numerical results and the analytical results.

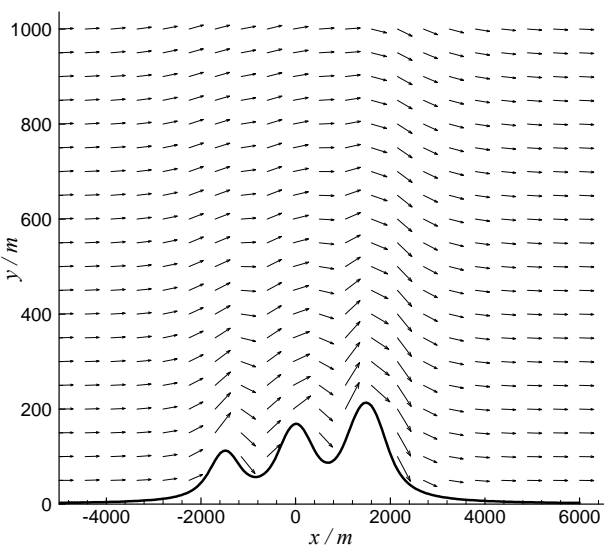

Figure 4. Wind velocity over the multiple mountains

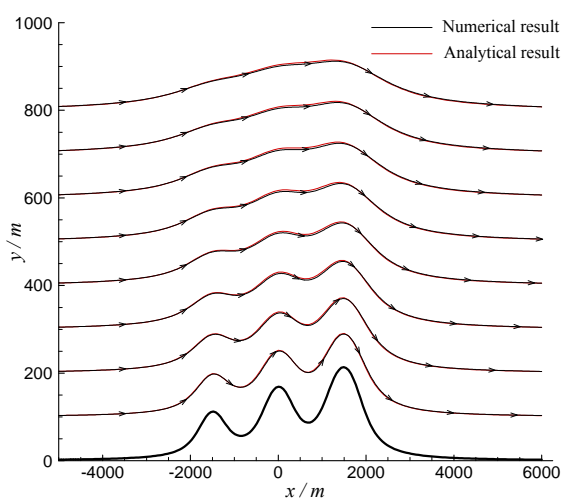

Figure 5. Streamline over multiple mountains

TABLE III. NUMERICAL RESULTS OF THE WIND VELOCITY THAT AFFECTED BY MULTIPLE MOUNTAIN

\begin{tabular}{|l|l|l|l|l|c|}
\hline \multicolumn{1}{|c|}{$\boldsymbol{x} / \boldsymbol{m}$} & \multicolumn{1}{c|}{$\boldsymbol{y} / \boldsymbol{m}$} & \multicolumn{1}{c|}{$\boldsymbol{V} / \boldsymbol{m} \boldsymbol{s}^{-\boldsymbol{I}}$} & $\boldsymbol{\theta}^{\boldsymbol{\rho}}$ & $\boldsymbol{\Delta} \boldsymbol{V}$ & $\boldsymbol{\Delta \theta}$ \\
\hline-3000 & 600 & 4.94 & 0.97 & $0.41 \%$ & $-3.23 \%$ \\
\hline-1500 & 600 & 5.17 & 1.39 & $0.35 \%$ & $-1.10 \%$ \\
\hline 0 & 600 & 5.32 & 1.02 & $0.27 \%$ & $4.81 \%$ \\
\hline
\end{tabular}

\begin{tabular}{|c|l|l|c|c|c|}
\hline $\boldsymbol{x} / \boldsymbol{m}$ & $\boldsymbol{y} / \boldsymbol{m}$ & $\boldsymbol{V} / \boldsymbol{m} \mathbf{s}^{-\boldsymbol{I}}$ & $\boldsymbol{\theta}^{\boldsymbol{o}}$ & $\boldsymbol{\Delta} \boldsymbol{V}$ & $\boldsymbol{\Delta \theta}$ \\
\hline 1500 & 600 & 5.58 & -0.85 & $0.30 \%$ & $-24.05 \%$ \\
\hline
\end{tabular}

Fig. $2 \sim$ Fig. 5 indicates that, the methods mentioned in [3] and [4] could only get the symmetrical terrain models. Compared with the analytical results, the numerical results obtained by the proposed method match it very well, the errors of the velocity magnitudes are all less than $1 \%$. And the absolute values of the error of the velocity direction are about $5 \%$. And the error could be decreased by increasing the elements number.

\section{Wind field over arbitrary terrains}

Because the ideal flow over a cylinder has a fixed type, the terrain shapes that could be simulated by this method are still limited, even the shape factor is used. Therefore, a lot of parameter adjusting work will be needed for the multiple cylinders superposition method to simulate an arbitrary terrain as shown in fig.1, and it has a pretty low efficiency.

For the terrains shown in fig. 1 , the sea level range is $x \in$ $[-\infty, 0]$, with a gentle slope at $x \in[0,1000]$, it transfers to the complex terrains at the range of $x \in[1000,4000]$. The plain range is $x \in[4000,+\infty]$. The wind field is defined as $x \in[-$ 2000,6000] , $y \in[0,2000]$. Velocity of uniform free stream is still $5 \mathrm{~m} / \mathrm{s}$. Using the straight line and cubic spline curve to describe this complex terrain shape, and the curve of terrain is divided into 400 elements. Fig. 6 and Fig. 7 shows the results of wind velocity and streamlines over the complex terrain.

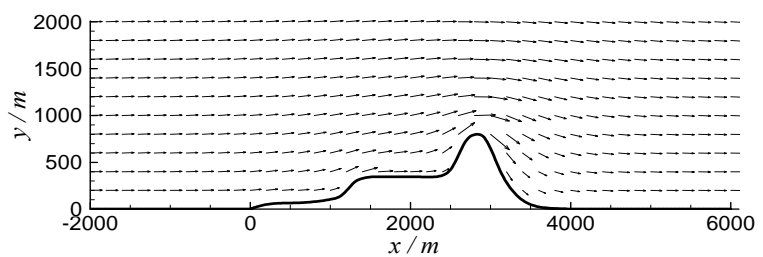

Figure $6 . \quad$ Wind field affected by the arbitrary terrain

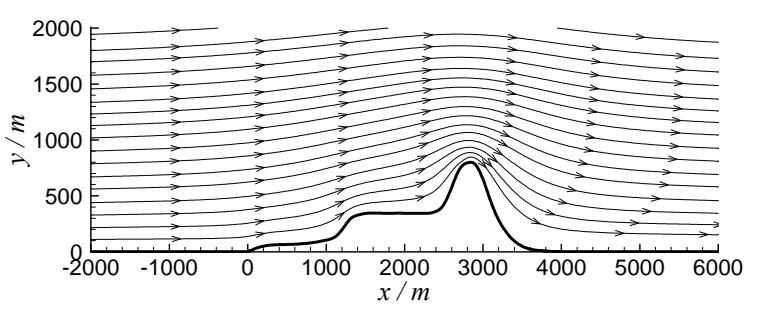

Figure 7. Streamline over the arbitrary terrain

\section{EXAMPLE}

To study the effects of wind field on airplane must be establish the flight vehicle motion equation in the nonquietude atmosphere [6]. In this paper, Based on the example of an airplane through this wind field, according to the above theories to set the flight vehicle motion equations of symmetrical plane in the non-quietude atmosphere, and discussing effect of complex terrain on flight path. 


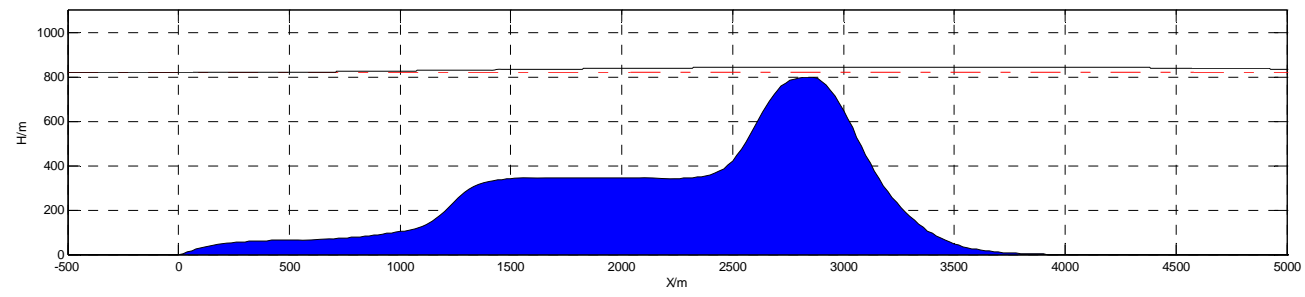

Figure 8. Contrast diagram between planned and actual track

Chosen flow velocity ahead is $5 \mathrm{~m} / \mathrm{s}$, balanced flight states is, $M_{a}=0.4, h=820 \mathrm{~m}$. Fig. 8 shows the contrast diagram between planned and actual track. Fig. 9 shows magnified schematic diagram of these two tracks. As can be seen from these graphs, in the terrain gentle areas, the terrain has minor effect on the track, and as terrain grows in complexity, the change of track is more apparent. Near the peaks, the change reaches the maximum offset, and then the offset is gradually reduced.

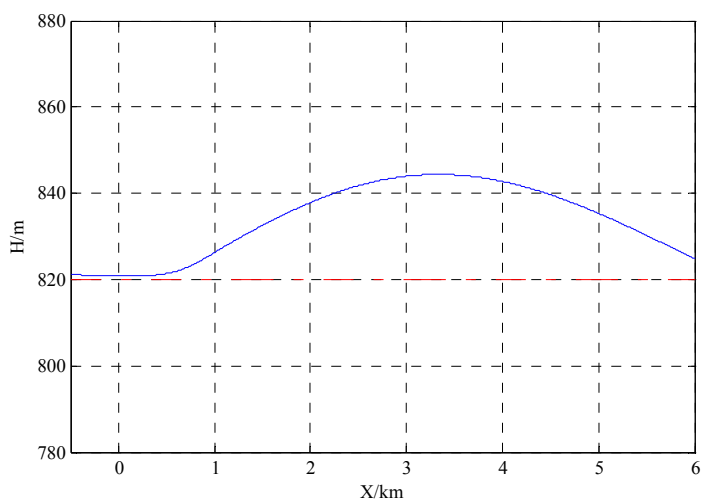

Figure 9. Magnified schematic diagram of these two tracks

\section{CONCLUSIONS}

Based on the aerodynamic potential flow theories, a numerical simulation method for two-dimensional airflow over complex terrains is developed in this paper. The effects of terrains on the wind field are considered by a serial of two-dimensional vortexes, and the vortex strengths are solved by combining with the ground boundary conditions. Numerical examples are studied by the proposed method, and the method is also evaluated by comparing the results with ones from the existing method. Conclusions are summarized below.

1) Complex terrain is represented by the combination of the straight lines and cubic spline curves, which could be defined by several predefined pass through points. The two- dimensional profile of complex terrains could be described by this method precisely. Compared with the analytical results of the airflow around a single cylinder, the numerical results obtained by the proposed method match the analytical results very well.

2) As a lot of parameter adjusting work will be needed for the multiple cylinders superposition method to simulate an arbitrary terrain, the computation procedure proposed in this paper is very simple and efficient, and could provide a wind field with considerable accuracy, which fits the requirements of engineering application very well. It could be used for flight principle evaluation and flight simulators.

3) Through analysis of flight path found that, terrain has a direct impact on track. In areas of complex terrain, flight path changes with terrain.

\section{ACKNOWLEDGMENT}

Thanks for the Funds Program: Joint Program for Scientific Research and Graduates Cultivation in Colleges and Universities in Beijing.

\section{REFERENCES}

[1] L. Clark Terry, G. Robert. Tree-dimensinal Numerical Model Simulations of Airflow Over Mountainous Terrain: A Comparsion with Observations. Monthly Weather Review. 1982, pp. 110.

[2] P. Hu, D. Chen, Y.H. Zheng, et al. Numerrical Simulation of Airflow over Three-Dimentional Moutains. Metoorology Journal of Henan. 1996.

[3] G.X. Hong, H.N.Dong, C.J. Jin. An Engineering Simulation Mode of Air flow over Mountain. Flight Dynamic. 1998,vol. 16, pp. 24-28.

[4] G. Liu, G.X. Hong, C.J. Jin. Engineering Simulation Method for Airfield over Complex Terrain at Minimum Altitude. Journal of Beijing University of Aeronautics and Astronautics. 2003, vol.29, pp. 193-196.

[5] Z. Yi, X. Wu, L.Z. Zhou. Low Speed Aerodynamics: Metallurgical Industry Press. Beijing, 2005.

[6] Y.L. Xiao, Vehicle Motion Equation, Aviation Industry Press, Beijing, 1987. 